\title{
Endoscopic Botulinum Toxin Injection for Tacrolimus-Induced Achalasia in a Renal Transplant Recipient
}

\author{
Marilia Campos ${ }^{\mathrm{a}, \mathrm{b}}$, Robert Matlock ${ }^{\mathrm{a}}$
}

\begin{abstract}
Post-transplantation achalasia secondary to immunosuppression is a rare complication. Here we present a man who developed dysphagia 4 days after his renal transplant and was found to have achalasia thought to be due to his tacrolimus. Switching from one calcineurin inhibitor to another was not possible in our patient due to the high risk of inducing rejection; thus he was successfully treated with the botulinum injection. To our knoledge, this is the first case of tacrolimusinduced achalasia ever reported in a renal transplant patient; it is also the first case in a transplant patient to be treated endoscopically with botulinum injection.
\end{abstract}

Keywords: Achalasia; Calcineurin inhibitor; Tacrolimus; Botulinum toxin injection

\section{Introduction}

Post-transplants patients on calcineurin inhibitors (CNIs) have a low risk of developing achalasia. This is a rare side effect but should be considered in post-transplant patients with newonset dysphagia. The proposed mechanism involves the inhibition of nitric oxide synthase by CNIs which leads to changes in esophageal motility. Thus far, there have been two cases of CNI-induced achalasia in the literature, and both were treated by switching between different CNIs. Here we present the third reported case of CNI-induced achalasia and the first one ever treated with botulinum injection.

\section{Case Report}

A 58-year-old man with a history of chronic kidney disease stage 4-5 secondary to focal segmental glomerulosclerosis was initially diagnosed based on biopsy at age 17 after present-

Manuscript submitted March 5, 2019, accepted March 25, 2019

aDepartment of Medicine, Hennepin Healthcare, Minneapolis, MN, USA ${ }^{\mathrm{b}}$ Corresponding Author: Marilia Campos, Department of Medicine, Hennepin Healthcare, 701 Park Ave. (G5), Minneapolis, MN 55415, USA.

Email: Marilia.camposulloa@hcmed.org ing with nephrotic syndrome. He received steroids for several years, then was lost to follow-up and re-presented with endstage kidney disease in 2017.

Transplant evaluation led to identification of a clinically significant antibody (positive flow cross-match), although confirmation testing (antihuman globulin cross-match) returned negative.

Based on the pretransplant evaluation, the decision was made to proceed with the transplant while altering his immunosuppression protocol from Standard to High Risk. This protocol included thyroglobulin induction and maintenance tacrolimus.

At clinic follow-up on postoperative day 4 , the patient reported difficulty in swallowing since postoperative day 2 . This progressed to dysphagia for solids, liquids and pills over the next few weeks. Of note, he had no dysphagia prior to his transplant.

A modified barium swallow study (Fig. 1) demonstrated severe esophageal dysmotility and lower esophageal sphincter dysfunction concerning for achalasia. Upper endoscopy (Fig. 2) 1 week later revealed a dilated esophagus with mild edema but without focal lesions or ulcerations. Biopsies from the stomach and esophagus were negative for gastritis, eosinophilia, citomegalovirus (CMV) and herpes simplex virus (HSV). Esophageal manometry (Fig. 3) evidenced outflow obstruction of the esophagogastric junction and weak peristalsis in the esophageal body, confirming the diagnosis of achalasia.

Although prior reports indicated that CNI-induced achalasia may resolve with a change in therapy [1], even within the same class of drug, this option was considered undesirable due to the patient's high risk for early transplant rejection. Similarly, surgical therapy for achalasia was considered too high risk in this patient so shortly after transplant. The patient ultimately underwent therapy with endoscopic botulinum toxin injection (100 units) of the lower esophageal sphincter. He had excellent results with immediate and complete resolution of dysphagia. Botulinum toxin has been used previously to treat achalasia [2], but this is the first time it has been used in tacrolimus-induced achalasia.

At his last clinical encounter, 8 months after his botulinum toxin injection, he remains free from dysphagia. Because the benefits of botulinum toxin injection in achalasia are generally temporary, repeat injections may be required, especially if a change in immunosuppression is still not advised based on the estimated risk of rejection.

\section{Discussion}

There are two previous reports of CNI-induced achalasia: one 


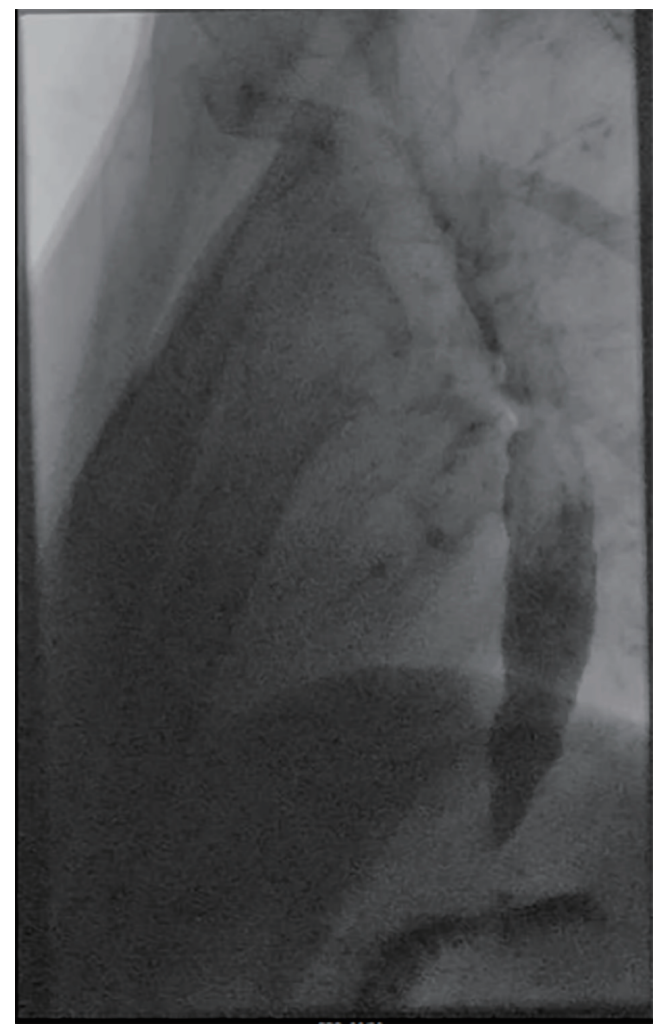

Figure 1. Barium swallow (March 27, 2018) with severe esophageal dysmotility and lower esophageal sphincter dysfunction concerning for achalasia.

following liver transplant while using cyclosporine and the other after allogenic hematopoetic stem cell transplant with use of tacrolimus [1]. To our knowledge, this is the first case reported after a kidney transplant, and the first successfully

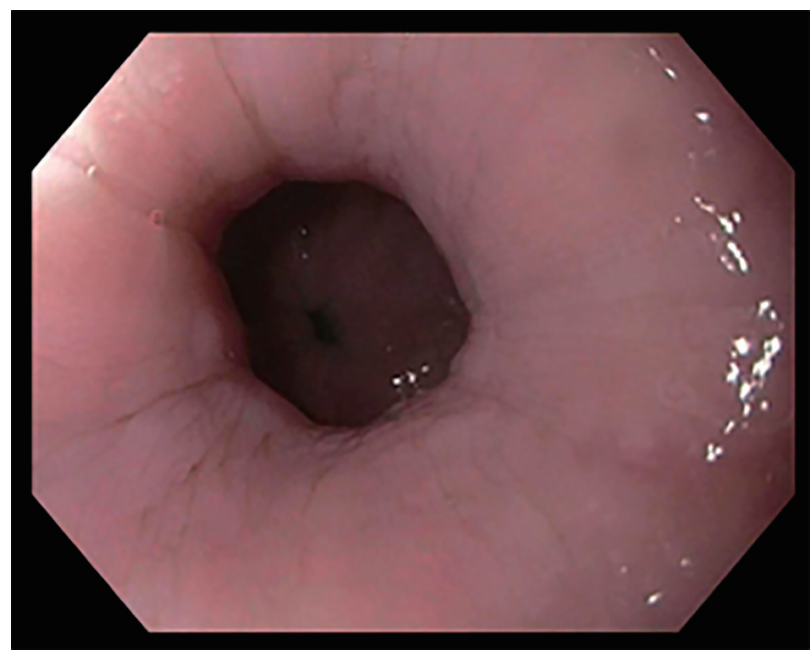

Figure 2. Upper endoscopy (EGD) (April 12, 2018) showing esophageal dilation.

treated with botulinum toxin injection. Similar to the current patient's experience, in both of the previously reported cases, dysphagia developed almost immediately following initiation of CNI therapy. These cases, however, were successfully managed with change in medical therapy to a different CNI.

The proposed mechanism for CNI-induced changes in esophageal motility involves inhibition of nitric oxide synthase by CNIs $[3,4]$. Nitric oxide (NO) is responsible for esophageal peristalsis as well as relaxation of the lower esophageal sphincter (LES). Decreases in NO levels in other diseases were associated with stiffening of the LES and dyssynchronous or absent esophageal peristalsis, as seen in achalasia [5]. Although it is unclear why switching between different CNIs would lead to symptom resolution, individual variation in the

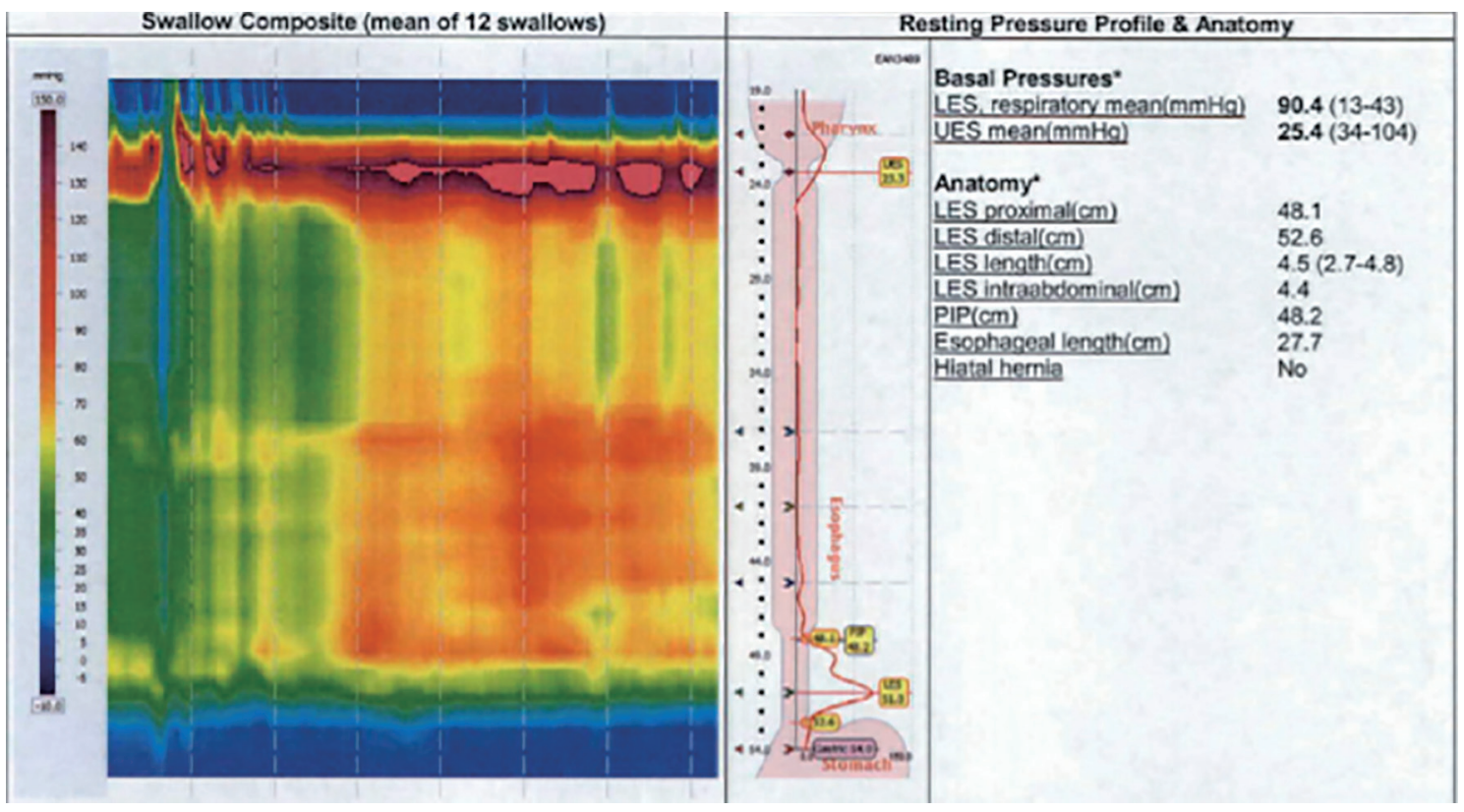

Figure 3. Esophageal manometry (May 17, 2018) demonstrating classic findings of achalasia. 
reaction to the different chemical structures of tacrolimus and cyclosporine has been proposed.

In the current patient, a change in therapy from tacrolimus to cyclosporine was considered prohibitively high risk due to the patient's risk of rejection following his transplant. His successful treatment with botulinum toxin injection is the first described successful endoscopic treatment for this rare condition.

\section{Conclusion}

Achalasia is an uncommon side effect of CNIs in the posttransplant population, but should be considered in patients with new onset dysphagia with otherwise negative workup. Esophagram may suggest the diagnosis, but clinical and EGD is required to rule out chemotherapy-related mucositis, gastrointestinal graft-versus-host disease (GVHD), CMV reactivation and reflux-related inflammation [5]. Ultimately, esophageal manometry serves as the gold standard diagnostic test.

This is the third reported case of CNI-induced achalasia, the first in a renal transplant recipient, and the first treated with botulinum toxin. Previous cases have been managed with a change in medical therapy. Botulinum toxin injection produced an excellent response with durable relief of symptoms more than 8 months following treatment. Botulinum toxin can be considered as a therapeutic option in transplant patients with the rare complication of CNI-induced achalasia.

\section{Acknowledgments}

We are grateful to Dr. Mark Linzer for comments on an earlier draft of this paper.

\section{Financial Disclosure}

None to declare.

\section{Conflict of Interest}

None to declare.

\section{Informed Consent}

Informed consent was obtained from the patient.

\section{Author Contributions}

Marilia Campos: writing of initial manuscript. Robert Matlock: proofreading and edits.

\section{References}

1. Koch R, Zoller H, Graziadei I, Propst A, Vogel W. Cyclosporine A-induced achalasia-like esophageal motility disorder in a liver transplant recipient: successful conversion to tacrolimus. Transplantation. 2003;76(4):744-745.

2. Vaezi MF, Pandolfino JE, Vela MF. ACG clinical guideline: diagnosis and management of achalasia. Am J Gastroenterol. 2013;108(8):1238-1249; quiz 1250.

3. Cook LG, Chiasson VL, Long C, Wu GY, Mitchell BM. Tacrolimus reduces nitric oxide synthase function by binding to FKBP rather than by its calcineurin effect. Kidney Int. 2009;75(7):719-726.

4. Hamalainen M, Lahti A, Moilanen E. Calcineurin inhibitors, cyclosporin A and tacrolimus inhibit expression of inducible nitric oxide synthase in colon epithelial and macrophage cell lines. Eur J Pharmacol. 2002;448(23):239-244.

5. Mashimo H, Goyal RK. Physiology of esophageal motility. https:/www.nature.com/gimo/contents/pt1/full/ gimo3.html. Accessed July 26, 2017. 